\title{
Research on production safety management of manufacturing industry based on improved HFACS

\author{
GaoQing
}

School of Economics \& Management, Jiangsu University of Science and Technology, Zhenjiang, 212003,China

1027369867@qq.com

Keywords: ABC behavior analysis, HFACS, set pair theory, human factor analysis.

\begin{abstract}
In the human-computer environment of the production system, the safety and reliability of the production equipment is increasing gradually with the increase of automation level, so the influence of people on the production system is more significant. In this paper, in view of the production safety problems in the manufacturing field, this paper improves the human factor analysis and classification (HFACS) model with the ABC behavior analysis method, and divides the unsafe factors into the 4 types of causes, and the 19 factors. Taking the M enterprise in Suzhou as an example, the weight of each factor is calculated by analytic hierarchy process, and the safety of the production system is analyzed and evaluated with the theory of set pair analysis. Finally, the countermeasures are put forward.
\end{abstract}

\section{基于改进的HFACS制造业生产安全管理研究一一M企业为例 \\ 高青 \\ 江苏科技大学经济管理学院，镇江，212003 \\ 1027369867@qq.com}

关键词: $A B C$ 行为分析法;HFACS; 集对理论;人因分析

中文摘要. 在生产系统人机环境中, 随着自动化水平的提高, 生产设备的安全可靠性逐渐增强, 所以人相对来说对生产系统的影响更为显著。本文针对制造业生产现场中的生产安全问题, 结合前因-行为-结果 (ABC) 行为分析法对人因分析与分类 (HFACS) 模型进行改进, 将生 产中的不安全因素分为 4 类致因, 19 类人因因素。以苏州市 $\mathrm{M}$ 企业为例, 通过层次分析法计 算各因素权重, 结合问卷调查情况, 运用集对分析理论对该生产系统安全性进行分析评价, 最终提出对策性建议。

\section{1. 引言}

我国的安全生产越来越受到重视, 很多专家学者对各行业的事故情况和安全管理进行深 入研究, 在事故的预防和从业人员的不安全行为等方面提出了建议, 但是针对制造生产企业 从业人员的不安全行为以及企业生产事故预防方面的研究目前还是比较少的。随着制造业企 业的系统自动化水平逐渐提高, 生产设备的可靠性、安全性逐步增强, 生产环境和氛围日渐 改善, 企业管理者对企业的管理和制度的制定更加科学和完善, 由于以上四个因素直接引发 
的事故逐渐减少, 而人作为整个生产活动的主体, 在生产系统中的影响更为突出和重要。因 此, 由于人的因素而导致的事故成为最主要的来源之一。

为提高企业安全管理水平、预防事故，本文采用事故致因理论对苏州市 $\mathrm{M}$ 制造企业的生 产安全问题进行研究，建立模型，以期找到改善的措施。

\section{2. 改进的HFACS模型}

HFACS（人因分析与分类系统）是Shappell等人在“瑞士奶酪”理论的基础上创建起来的 ${ }^{[1,2]}$ 。该模型归纳了导致事故发生原因的4个层次，分别为组织影响、不安全监管、不安全行 为前提和不安全行为。这4个层次因素是由组织影响开始的, 在一系列的反应后导致不安全行 为, 最终导致事故的发生。其中, 不安全行为属于显性因素, 直接导致事故发生; 而组织影 响、不安全行为的监督、不安全行为的前提属于隐性因素, 是事故发生的潜在来源。然而如 果其中有 1 个层次因素被制止，事故就不会发生。

HFACS模型最早应用于航空领域, 所以不适合直接应用到制造业。研究表明, 人的不安 全行为引起的事故占 $90 \%$ 以上，因此企业的安全管理的重点就是对人的不安全行为的控制。 不安全行为的定义是指在操作过程中, 正常行为受到来自内在和外在原因的干扰行, 偏离正 常轨迹产生的不符合操作规范的偏差性行为 ${ }^{[3]}$ 。国外Philip等人运用 $\mathrm{ABC}$ 行为分析法对不安全 行为进行研究 ${ }^{[4]}, A$ (Activator)指行为发生的原因; $B$ (Behavior)指行为; $C$ (Consequence)指行为 产生的结果以及对行为产生的反馈信息。它们三者相互作用相互影响。将 $\mathrm{ABC}$ 分析法应用于 HFACS模型中, 把组织影响、不安全监管、不安全行为的前提三者作为前因(A), 引发不安全 行为的产生; 不安全行为作为工人生产过程中的行为(B); 生产事故作为结果 $(\mathrm{C})$, 由工人的 不安全行为导致, 并且结果会对不安全行为的前提和不安全行为产生反馈作用。通过对 $\mathrm{M}$ 企 业2015年 2017年间生产安全报告的查阅整理，运用访谈法对M企业生产部、生产技术支持部 以及质量部相关人员进行访谈, 以及运用观察法对生产现场进行观察, 结合 $\mathrm{M}$ 企业实际生产 情况, 对HFACS模型做出改进, 从而得出新的HFACS框架。改进后的制造业HFACS模型如图 1所示，共包括4类事故致因、19个人因因素。其中事故致因人因因素及释义如表1所示。

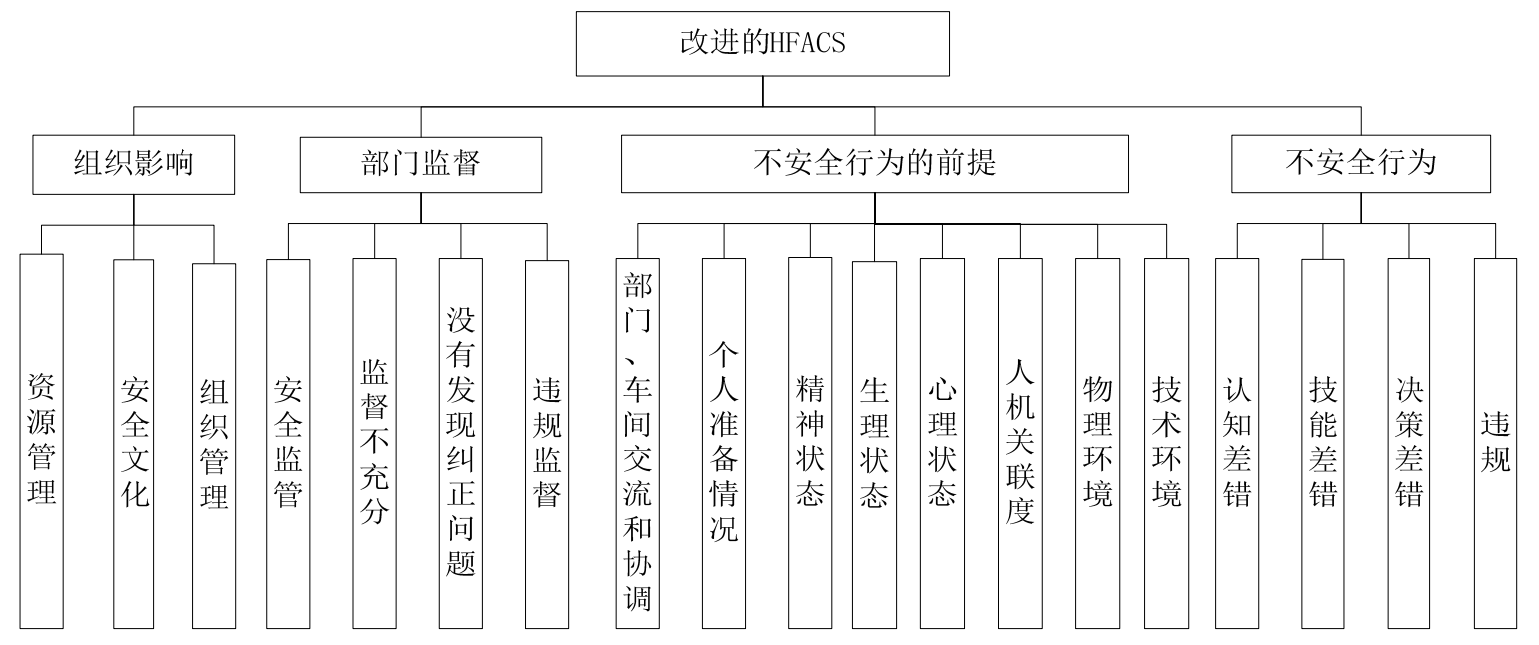

图 1 改进的 HFACS 模型

表 1 事故致因因素及释义

\begin{tabular}{|c|l|}
\hline 因素 & \multicolumn{1}{|c|}{ 释义 } \\
\hline 组织管理 & 上层决策失误; 责任落实不到位; 班组安排有缺陷; 规程及应急预案不完善; \\
\hline 安全文化 & $\begin{array}{l}\text { 管理层结构缺陷; 培训不到位; 员工安全知识掌握不到位; 厂房安全氛围不足; } \\
\text { 不重视安全等 }\end{array}$ \\
\hline 资源管理 & $\begin{array}{l}\text { 组织资源分配不当; 安全设备不到位; 安全管理人员不足; 人员使用不当; 安 } \\
\text { 全经费过度削减等 }\end{array}$ \\
\hline
\end{tabular}




\begin{tabular}{|c|c|}
\hline 安全监管 & 监管部门检查力度不够; 规章制度和应急预案完善与否; \\
\hline 监督不充分 & 监督政策缺失; 无监督培训; 现场管理有漏洞等; \\
\hline 没有发现、纠正问题 & 违规操作; 危险行为; 未及时检查设备; 不妥当制度条例未及时修改等; \\
\hline 违规监督 & 监督制度不合理；监督执行力度不够；无证上岗等; \\
\hline 部门、车间交流和协调 & 生产安排信息沟通不畅; 互相包庇、盯岗缺少; 相互协作等 \\
\hline 个人准备情况 & 技能素质训练不足; 未遵循休息的要求; \\
\hline 精神状态 & 情景意识较低; 安全意识不强; 精神劳累等 \\
\hline 生理状态 & 身体不适; 身体缺陷; 体能不适应等 \\
\hline 心理状态 & 不良心理, 如侥幸心理、省能心理、经验心理、逞能心理、厌倦心理等 \\
\hline 人机关联度 & 没有防护设备；设备缺陷，检修不到位; \\
\hline 生产工艺 & 产品生产步骤错误；生产条件不充分；设备布局不合理等; \\
\hline 物理环境 & 高温；高压；照明；湿度；噪音；辐射等; \\
\hline 认知差错 & 认知与实际情况不符; 认知和理解上有差错; \\
\hline 技能差错 & 技能素养不够; 操作步骤有误; 缺少专业培训等; \\
\hline 决策差错 & 计划制定不充分；经验不足；危险辨识不足； \\
\hline 违规 & 习惯性违规；偶然性违规; \\
\hline
\end{tabular}

\section{3. 集对分析}

\section{1 集对理论介绍}

集对理论由我国学者赵克勤提出 ${ }^{[5]}$, 该理论是一种分析事物之间不确定性关联度的分析 方法。应用该方法可以很好地评价企业人因分析与分类系统, 找出引发企业生产事故的显著 影响因素。集对分析包含三种联系, 包括同一性联系、对立性联系以及差异性联系。对于某 一具体问题下，两个不同集合的联系度表达式为:

$$
\mu=\frac{S}{N}+\frac{F}{N} i+\frac{P}{N} j=a+b i+c j
$$

上式中, $\mu$ 表示的是两个不同集合之间的关联度，范围是 $[-1,1] ; N$ 表示的是研究集对 中两个集合所有的特性总数; $S$ 表示的是两个集合共有的特性数; $P$ 表示的是两个集合中相 互对立的特性数; $F=N-S-P$ ，表示的是既不相互共有又不相互对立的特性数。 $a=S / N, b=F / N, c=P / N$, 分别表示为集对的同一度、差异度和对立度。由定义可知: $a+b+c=1$ 。 $i$ 表示的是差异度系数, 取值范围 [-1,1]; $j$ 表示为对立系数, 一般情况下取 值为 -1 。考虑权重时, 联系度表达式可写成如下形式:

$$
\mu=a+b i+c j=\sum_{k=1}^{S} \omega_{k}+\sum_{k=S+1}^{S+F} \omega_{k} \mathrm{i}+\sum_{k=S+F+1}^{N} \omega_{k} \mathrm{j}=W R E=\left(\omega_{1}, \omega_{2}, \ldots, \omega_{n}\right)\left(\begin{array}{c}
a_{1} b_{1} c_{1} \\
a_{2} b_{2} \\
c_{2} \\
\ldots \ldots \\
a_{n} b_{n} c_{n}
\end{array}\right)\left(\begin{array}{c}
1 \\
i \\
j
\end{array}\right)
$$

在上式中, $\omega_{k}$ 为特性的权重。当 $i=-1$ 时, 不确定度则转化为对立度; 当 $i$ 取值在 $[-1,1]$ 之 间时，则不确定度中既存在同一又存在对立。 


\section{2 集对势}

集对势用 $e$ 表示，是指当联系度中的对立度 $c \neq 0$ 时，同一度 $a$ 和对立度 $c$ 的比值。记作 $e=a / c$ 。同势表明两个集合存在同样的趋势, 反势表明两个集合存在对立的趋势, 均势表 明两个集合趋势比较平均。通过集对势的计算，可以确定系统的不确定性程度。

运用集对势来反映企业生产系统风险大小。将 $a$ 表示安全值, 即风险可接受的范围; $c$ 表示危险; 不确定部分用 $b_{i}$ 表示。随着 $i$ 取值的变化, 企业的安全性也会相应变化。企业 的安全势范围在最大安全势和最小安全势之间。当 $b+c \neq 0$ 时, 悲观势表达式为 $s h i(B)=\frac{a}{b+c}$ 。 $\operatorname{sh} i(B)>1$ 为安全状态, $\operatorname{sh} i(B)<1$ 为危险状态。

基于以上理论，对该企业人因因素进行集对分析后，其安全等级应为奇数个等级，根据 均分原则将其分为 3 级: 危险、一般、安全。3个安全等级的范围分别是: 危险 $-1 \leq \mu \leq-0.33$ 、 一般 $-0.33<\mu \leq 0.33$ 、安全 $0.33<\mu \leq 1$ 。

\section{4. 应用分析}

以M 企业人因事故HFACS 模型分析为例, 通过多位专家对指标评价构建判断矩阵, 运 用层次分析法计算各指标权重，见表 2 。计算得 $C R=0<0.1$ ，通过一致性检验，结果可靠。

采用调查问卷的形式对该厂进行等级评定，向该企业生产管理部、生产技术支持部、质 量部以及车间工人了 200 份问卷。问卷中针对模型中19项指标均有“良好（安全）”、“一般”、 “差（危险）”3个选项，分别对应集对分析模型中的“同、异、反”3 种情况。

运用SPSS 对调查问卷进行信度检验, Cronbach $\alpha=0.908$, 说明数据信度质量较高, 可用于进一步分析。归一化处理后得到矩阵R。

表 2 各指标权重及统计指标

\begin{tabular}{|c|c|c|c|c|c|c|c|c|c|c|}
\hline \multirow{2}{*}{ 事故致因 } & \multirow{2}{*}{$\begin{array}{l}\text { 权 } \\
\text { 重 }\end{array}$} & \multirow{2}{*}{ 因素 } & \multirow{2}{*}{ 权重 } & \multirow{2}{*}{$\begin{array}{c}\text { 总权 } \\
\text { 重 } \\
\end{array}$} & \multicolumn{3}{|c|}{ 统计数据 } & \multicolumn{3}{|c|}{ 归一数据 } \\
\hline & & & & & 安全 & 一般 & 危险 & 安全 & 一般 & 危险 \\
\hline \multirow{3}{*}{ 组织影响 } & \multirow{3}{*}{0.28} & 组织管理 & 0.4 & 0.112 & 156 & 28 & 16 & 0.78 & 0.14 & 0.08 \\
\hline & & 安全文化 & 0.35 & 0.098 & 132 & 44 & 24 & 0.66 & 0.22 & 0.12 \\
\hline & & 资源管理 & 0.25 & 0.07 & 162 & 28 & 10 & 0.81 & 0.14 & 0.05 \\
\hline \multirow{4}{*}{$\begin{array}{c}\text { 不安全监 } \\
\text { 督 }\end{array}$} & \multirow{4}{*}{0.21} & 安全监督 & 0.36 & 0.0756 & 170 & 30 & 0 & 0.85 & 0.15 & 0 \\
\hline & & 监督不充分 & 0.13 & 0.0273 & 158 & 32 & 10 & 0.79 & 0.16 & 0.05 \\
\hline & & 未发现纠正问题 & 0.35 & 0.0735 & 174 & 20 & 6 & 0.87 & 0.03 & 0.03 \\
\hline & & 违规监督 & 0.16 & 0.0336 & 170 & 22 & 8 & 0.85 & 0.04 & 0.04 \\
\hline \multirow{8}{*}{$\begin{array}{c}\text { 不安全行 } \\
\text { 为前提 }\end{array}$} & \multirow{8}{*}{0.34} & $\begin{array}{c}\text { 部门、车间交流 } \\
\text { 和协调 }\end{array}$ & 0.1 & 0.034 & 156 & 34 & 10 & 0.78 & 0.05 & 0.05 \\
\hline & & 个人准备情况 & 0.1 & 0.034 & 164 & 20 & 16 & 0.82 & 0.08 & 0.08 \\
\hline & & 精神状态 & 0.18 & 0.0612 & 136 & 44 & 20 & 0.68 & 0.1 & 0.1 \\
\hline & & 生理状态 & 0.12 & 0.0408 & 138 & 50 & 12 & 0.69 & 0.06 & 0.06 \\
\hline & & 心理状态 & 0.15 & 0.051 & 150 & 42 & 8 & 0.75 & 0.04 & 0.04 \\
\hline & & 人机关联度 & 0.08 & 0.0272 & 156 & 30 & 14 & 0.78 & 0.07 & 0.07 \\
\hline & & 生产工艺 & 0.15 & 0.051 & 164 & 20 & 16 & 0.82 & 0.08 & 0.08 \\
\hline & & 物理环境 & 0.12 & 0.0408 & 142 & 38 & 20 & 0.71 & 0.19 & 0.1 \\
\hline \multirow{4}{*}{$\begin{array}{c}\text { 不安全行 } \\
\text { 为 }\end{array}$} & \multirow{4}{*}{0.17} & 认知差错 & 0.2 & 0.034 & 174 & 16 & 10 & 0.87 & 0.08 & 0.05 \\
\hline & & 决策差错 & 0.28 & 0.0476 & 174 & 16 & 10 & 0.87 & 0.08 & 0.05 \\
\hline & & 技能差错 & 0.3 & 0.051 & 166 & 22 & 12 & 0.83 & 0.11 & 0.06 \\
\hline & & 违章 & 0.22 & 0.0374 & 170 & 24 & 6 & 0.85 & 0.12 & 0.03 \\
\hline
\end{tabular}


将 $\boldsymbol{W}$ 与 $\boldsymbol{R}$ 带入，可得 $\mu=0.7866+0.1509 i+0.0625 j$, 即同一度 $a=0.7866$, 差异度 $b=0.1509$, 对立度 $c=0.0625 \circ a>c, a>b>c$, 弱同势。根据得到的数据对 $\mathrm{M}$ 企业进行分析。

（1）集对势分析：由联系度表达式可知 $\mathrm{M}$ 企业集对势为弱同势，以同一趋势为主，同一 趋势较弱。同一度 $a=0.7866$ 表明该企业评价指标为“较好”, 差异度 $b=0.1509$ 表明部分指标 有变坏的趋势, 因此, 需要将表2中部分指标进行改进, 避免趋势恶化甚至发生事故。

（2）联系度分析：综合来看, 此企业联系度 $\mu \in[0.5732,0.875]$ ，处于“安全”等级，表 明该企业安全方面人因管理做到了较好，某些人因因素需要改善，防止发生事故。

(3) 不确定性分析：差异度 $b$ 决定该系统的不确定性， $b$ 值越大不确定性越大。该企业 $b=0.1509$, 表明该企业不确定性较高, 但总体来看, 安全状况较好。

(4) 悲观势分析：由联系度公式知，悲观势 $\operatorname{shi}(B)=a /(b+c)=0.7866 /(0.1509+0.0625)=3.686>1$, 处于“安全”级别。

\section{5. 结论}

本文基于 $\mathrm{ABC}$ 行为分析方法对 $\mathrm{M}$ 企业的生产现场进行观察, 以此为依据建立HFACS模 型, 并通过“观察不安全行为一找出不安全行为的原因一及时纠正原因一再观察一继续找原 因”的不断循环，逐步纠正工人的不安全行为，为解决人机系统管理问题的提出了新思路。其 次, 应用改进的HFACS模型, 全面而细化总结工人不安全行为的影响因素。在数据分析方面, 应用了赵克勤学者提出的集对理论。集对理论将确定性和不确定性统一在同异反表达式中, 有效地对 $\mathrm{M}$ 企业引起人的不安全行为原因进行确定性分析和不确定性分析, 更能系统、定量 地对制造业安全管理提出合理建议。

\section{References}

[1] Scott A. Shappell, Douglas A. Wiegmann. The Human Factors Analysis and Classification System-HFACS[J]. American Libraries, 2000, 1(1):20-46.

[2] Yan-Bin L I, Jin N, Hong M L. Human accident hidden hazard identification and evaluation of power grid enterprise based on HFACS and grey correlation analysis method[J]. Journal of Safety Science \& Technology, 2013, 9(2):157-161.

[3] LI Lei,Research on formation mechanism and combination intervence of coal miners' unsafe behaviors[D]Xi'an University of Science and Technology ,2014

[4] Lehman P K, Geller E S. Behavior analysis and environmental protection: Accomplishments and potential for more.[J]. Behavior \& Social Issues, 2005, 13(1).

[5] Zhao K, Jiang Y. Primary Discussion on Some Systematical and Dialectical Thinking in Set Pair Analysis[J]. Journal of Systemic Dialectics, 2000. 